\title{
HPLC and high-throughput sequencing revealed differences of tea-leaves quality components and soil microbial community structure between modern and ancient tea plantations in Lincang Region
}

\author{
Guangrong Yang \\ Yunnan Agricultural University \\ Dapeng Zhou \\ Yunnan Agricultural University \\ Renyuan Wan \\ Yunnan Agricultural University \\ Conglian Wang \\ Yunnan Agricultural University \\ Jin Xie \\ Yunnan Agricultural University \\ Cunqiang Ma ( $\sim$ macunqiang1208@aliyun.com ) \\ Yunnan Agricultural University \\ Yongmei Li \\ Yunnan Agricultural University
}

\section{Research Article}

Keywords: Ancient tea plantation, Amino acids, Phenolic components, Soil fertility, Microbial community structure

Posted Date: January 12th, 2022

DOI: https://doi.org/10.21203/rs.3.rs-1185277/v1

License: (c) (i) This work is licensed under a Creative Commons Attribution 4.0 International License. Read Full License 


\section{Abstract}

\section{Background}

Ancient tea plantations with an age over 100 years still reserved at Mengku Town in Lincang Region of Yunan Province, China. However, the characteristic of soil chemicophysical properties and microbial ecosystem in the ancient tea plantations and their impact on tea-leaves chemical components remained unclear. Tea-leaves chemical components including amino acids, phenolic compounds and purine alkaloids, and soil chemicophysical properties including $\mathrm{pH}$, cation exchange capacity (CEC), soil organic matter (SOM), soil organic carbon (SOC), total total nitrogen (TN), total phosphorus (TP), total potassium (TK), alkali-hydrolyzable nitrogen (AN), available phosphorous (AP) and available potassium (AK), and microbial community structure of modern and ancient tea plantations in five geographic sites (i.e. Bingdao, Baqishan, Banuo, Dongguo and Jiulong) were determined by high performance liquid chromatography (HPLC) and high-throughput sequencing, respectively.

\section{Results}

Tea-leaves chemical components, soil chemicophysical properties and microbial community structures including bacterial and fungal community abundance and diversity evaluated by Chao 1 and Shannon varied with geographic location and tea plantation type. The ancient tea plantations possessed significantly $(P<0.05)$ higher free amino acids, gallic acid, caffeine and EGC in tea-leaves, as well as soil fertility. The bacterial community structure kept stable, while fungal community abundance and diversity significantly $(P<0.05)$ increased in ancient tea plantation because of higher soil fertility and lower $\mathrm{pH}$. The long-term plantation in natural cultivation way significantly $(P<0.05)$ improved the abundances of Nitrospirota, Methylomirabilota, Ascomycota and Mortierellomycota phyla.

\section{Conclusions}

Due to the natural cultivation way, the ancient tea plantations still maintained relatively higher soil fertility and complete soil microbial ecosystem, which contributed to the sustainable development with higher quality in tea-leaves.

\section{Background}

Tea (Camellia sinensis (L.) O. Kuntze) has become one of the most popular and widely consumed beverages in the world because of its multiple health benefits [1-3]. Southwest China including Yunan, Guizhou and Sichuan Province have been regarded as the origin center, which is now widely cultivated all over the world for approximately 5,000 years [4-6]. Until now, Lincang, Puer and Xishuangbanna Regions in Yunnan Province, China, still remained abundant ancient tea trees of wild, transitional and artificial culture types, respectively [7-8]. In particular, the fresh tea-leaves collected from the artificial culture trees of ancient tea plantations with an age over 100 years could be processed for Pu-erh tea, black tea and white tea [8]. 
Processing technology, tea cultivars, production places and seasons significantly impacted phenolic compounds including catechins (a subgroup of flavan-3-ols), phenolic acids, flavonoids and anthocyanins, purine alkaloids, free amino acids like theathine, glutamate and arginine, and flavoalkaloids [9-11]. Additionally, compared to the ecological tea-leaves collected from modern tea plantations, white teas from ancient tea plantations has been confirmed with higher ameliorative effect on kidney damage in diabetic mice via Sirtuins 1/AMP-activated protein kinase (SIRT1/AMPK) pathway, while Pu-erh teas from ancient tea plantations are discovered to possess higher contents of amino acids, fatty acids, phenolic acids, nucleosides and nucleobases, and lower contents of flavonoids and caffeine congeners [12-13]. Generally, the fresh tea-leaves from ancient tea plantations showed relatively higher contents of tea polyphenols, total catechins, free amino acids, gallic acid, soluble sugars and caffeine, but significant $(P<0.05)$ lower polyphenols/amino acids ratio and ester catechins content, especially epigallocatechin gallate (EGCG). Due to the lower yields, much more nutrients absorbed by deeper roots and greater chemical profile variations, the tea-leaves collected and processed from ancient tea plantations are widely recognized to have better qualities and preserving values with a higher price [14].

The 15 rare earth elements contributed to the identification of ancient tea-leaves, which should be attributed to the soil nutrients and ecological system in ancient tea plantations [15]. As the supporter for survival and growth of tea trees, soil nutrients impacted the output and quality of tea-leaves, as well as microbial community composition [16]. In addition, the microbial including bacterial and fungal communities in rhizospheric soil play vital roles in ecological processes, such as soil organic matter (SOM) decomposition, soil nutrient cycling, and soil structure formation and stability [17-18]. Previous studies have indicated the variation of fungal community in rhizosphere soil with plantation age, and found the significantly decrease of soil bacterial community in modern tea plantations after 23 years cultivation, which potentially caused the decrease of glomalin-related soil protein and soil organic carbon (SOC) after long-term cultivation [19-20]. Unfortunately, the distribution and characteristic of tea-leaves quality components, soil chemicophysical properties and microbial ecosystem in the ancient tea plantations remain unclear.

Thus, in this study, we evaluated the main quality components of the fresh tea-leaves, and soil chemicophysical properties and microbial community in ancient and modern tea plantations from five geographical sites, which are all located at Mengku Town of Shuangjiang autonomous County in Lincang Region of Yunan Province, China. Based on high performance liquid chromatography (HPLC) and highthroughput sequencing, this study revealed the variations of tea-leaves quality components, soil chemicophysical properties and microbial community structure with the five geographical sites, and confirmed that tea-leaves from ancient tea plantations contained significantly $(P<0.05)$ higher free amino acids, caffeine, gallic acid and EGC contents, but significantly $(P<0.05)$ lower GCG and EGCG contents. Compared with modern tea plantations, the ancient tea plantations possessed significantly $(P<0.05)$ higher soil fertility, including CEC, SOM, SOC, AN, AP and AK, as well as significantly $(P<0.05)$ higher fungal community abundance and diversity (including Chao 1 and Shannon), while bacterial and actinomycetes community kept stable. Through comparisons, this study elaborated the sustainable development of ancient tea plantations from soil fertility and microbial community structure. 


\section{Materials And Methods}

\section{Experimental sites}

The study is performed at representative tea planting area that Mengku Town of Shuangjiang

autonomous County in Lincang Region of Yunan Province, which belongs to the subtropical mountain monsoon climate. The annual average temperature is $18^{\circ} \mathrm{C}$, and the annual average precipitation is from $1700 \mathrm{~mm}$ to $1900 \mathrm{~mm}$. The soil type is mainly laterite and yellow loam with the sand grains from granite sandy conglomerate and purple shale. Five sampling sites located at Mengku Town including Bingdao, Banuo, Baqishan, Dongguo and Jiulong all grow ancient tea plantations with an age over 100 years and modern tea plantations with an age below 50 years, respectively, which are present in Fig. 1. All tea plantations are grown a single cultivar of tea plant (Camellia sinesis var. assamica cv. Mengku Dayecha) and cultivated in accordance with the requirement of ecological tea garden that there were no farming, no fertilizers and no pesticides throughout the whole growing period.

\section{Soil collection and treatment}

The fallen leaves, weeds and stones on the surface of the soils from five subplots $(0.5 \times 0.5 \mathrm{~m})$ in every plot $(2 \times 2 \mathrm{~m})$ were clean up for the soil sampling collection. The soil samples were collected at the same cleared position. Sterile containers were used to store five soil samples at the $0-20 \mathrm{~cm}$ depth from five subplots in every plot, and these soil samples were blended into a composite soil sample. The field-moist clods $\left(<5 \mathrm{~mm}\right.$ ) underwent cool-drying at $4{ }^{\circ} \mathrm{C}$ until the gravimetric water concentration of about $80 \mathrm{~g} \mathrm{~kg}^{-1}$ were separated through different sieve mesh including $1 \mathrm{~mm}, 0.25 \mathrm{~mm}$ and $0.15 \mathrm{~mm}$ for the determinations of soil physicochemical properties. The field-moist clods collected at $0-4^{\circ} \mathrm{Cand}-86^{\circ} \mathrm{C}$ in aseptic condition were prepared for the determinations of microbiological quantification by culture medium and microbial community diversity by high-throughput sequencing, respectively.

\section{Tea-leaves sample collection}

The spring fresh tea-leaves with one bud and two leaves from different tea plantations were collected at the same period with reference to the method of Liu et al. (2020)[21]. The fresh tea-leaves were fixated by a $800 \mathrm{~W}$ microwave oven for $60-80 \mathrm{~s}$, and then dried by sunlight until the moisture content below $6.0 \%$ by dry weight. The dried tea-leaves were maintained at $-20^{\circ} \mathrm{C}$ for the chemical analysis, including tea polyphenols, free amino acids, 6 catechins, caffeine and gallic acid.

\section{Determinations of main chemical components in tea-leaves}

Moisture content and water extracts content were developed according to the national standard methods established by China National Institute of Standardization. Content of tea polyphenols was determined by a UV-1800PC ultraviolet-visible (UV-Vis) spectrophotometer (AOE Instruments, Shanghai, China) using Foline-Ciocalteu assay on $765 \mathrm{~nm}$ with gallic acid as the standard [22]. Total content of free amino acids was determined on $570 \mathrm{~nm}$ by the UV-Vis spectrophotometer using ninhydrin assay[22]. 
6 Catechins including catechin (C), epicatechin (EC), epigallocatechin (EGC), epigallocatechin gallate (ECG), gallocatechin gallate (GCG) and epigallocatechin gallate (EGCG), caffeine and gallic acid contents were determined by high-performance liquid chromatography (HPLC) through an Agilent 1200 series HPLC system (Agilent Technologies, Santa Clara, CA, USA) composed of diode array detector, infinity binary pump, integrated vacuum degasser, autosampler and thermostated column compartment and TSK gel ODS-80TM chromatogram column $(250 \mathrm{~mm} \times 4.6 \mathrm{~mm}, 5 \mu \mathrm{m}$; Tosoh Corporation, Tokyo, Japan) [23]. Solvent A ( $0.05 \mathrm{~mol} \mathrm{~L}^{-1}$ phosphoric acid water solution and $5 \%$ acetonitrile) and solvent $\mathrm{B}(0.05 \mathrm{~mol} \cdot \mathrm{L}-1$ phosphoric acid water solution and $80 \%$ acetonitrile) were prepared for HPLC separation. The gradient was programmed as follows:from 0-30 min, solvent A was decreased from $91 \%$ to $47 \%$, solvent B was increased from $9 \%$ to $53 \%$ with a flow rate of $0.7 \mathrm{~mL} \mathrm{~min}^{-1}$. The column temperature and detection wavelength were set at $30^{\circ} \mathrm{C}$ and $280 \mathrm{~nm}$, respectively. Additionally, the injection volume was $5 \mu \mathrm{L}$. Quantitative analysis was carried out based on the calibration curves of 8 standards. Quantitative analysis was carried out based on the calibration curves of 8 standards. Each tea-leaves sample was determined with 5 replications.

\section{Determinations of soil physicochemical properties}

The soil $\mathrm{pH}$ value was measured by glass recombination electrode method in soil: water solution =1:2.5 $(\mathrm{w} / \mathrm{w})$. The cation exchange capacity (CEC) content was determined by ammonium acetate exchange extraction-distillation method. The soil organic carbon (SOC) content was determined by an UV-Vis spectrophotometer method through potassium dichromate with glucose as the standard. The soil organic matter (SOM) content was measured by potassium dichchromate capacity method-external heating method. The total nitrogen (TN) content was measured by kjeldahl determination after digestion of concentrated sulfuric acid and hydrogen peroxide. The total phosphorous (TP) content was determined by molybdenum antimony anti colorimetric method after digestion of concentrated sulfuric acid and hydrogen peroxide. The total potassium (TK) content was determined by flame photometry after the digestion. The alkali-hydrolyzable nitrogen (AN) content was measured using alkalescent diffusion method of $0.5 \mathrm{~mol} / \mathrm{L} \mathrm{NaOH}$ solution. The available phosphorous (AP) content was determined through phosphomolybdate blue spectrophotometry method after the extract of $0.5 \mathrm{~mol} / \mathrm{L} \mathrm{NaHCO}$ solution. The available potassium (AK) content was measured by flame photometry after the extract of ammonium acetate. Each soil sample was determined with 5 replications.

\section{Determinations of microbial quantity in soil}

The microbial quantity including fungi, bacteria and actinomycetes were determined by dilution coating method through the relevant culture mediums, and the colony forming units (CFU) were calculated by per gram of dried soil [24]. Beef extract-peptone medium was carried out to measure bacterial quantity after 3 days of cultivation at $28^{\circ} \mathrm{C}$, while fungi number was measured through Martin's rose bengal medium after 5 days of cultivation at $28^{\circ} \mathrm{C}$. And the actinomycetes in soil were determined by Gao's No. 1 medium after 7 days of cultivation at $28^{\circ} \mathrm{C}$. 


\section{Soil bacterial and fungal community structure analyses}

The $0.5 \mathrm{~g}$ of field-moist clods was used to extract DNA through SDS lysis buffer - guanidinium isothiocyanate- polyethylene glycol (SDS-GITC-PEG) method [25]. The extracts were evaluated in terms of both quantity and quality through a NanoDrop ND-2000 spectrophotometer (NanoDrop, Wilmington, DE, USA). The primers 338F (5'-ACTCCTACGGGAGGCAG-3') and 806R (5'-GGACTACHVGGGTWTCTAAT-3') were selected to amplify the target $\mathrm{V} 4$ hypervariable regions of bacterial 16S rRNA genes for bacterial community structure analysis, while the primers ITS3F (5'-GCATCGATGAAGAACGCAGC-3') and ITS4R (5'TCCTCCGCTTATTGATATGC-3') were used for the amplification of the target V3-V4 hypervariable regions of fungal 18S rRNA genes for fungal community structure analysis [26-27]. The final volume of $20 \mu \mathrm{L}$ included $4 \mu \mathrm{L}$ of $5 \times$ TransStart FastPfu buffer, $2 \mu \mathrm{L}$ of dNTPs $(2.5 \mathrm{mM}), 0.5 \mu \mathrm{L}$ of Taq polymerase, $0.8 \mu \mathrm{L}$ of each primer $(5 \mu \mathrm{M}), 1.0 \mathrm{ng}$ of containing template DNA and $0.4 \mu \mathrm{L}$ of TransStart FastPfu DNA polymerase were used to implement amplifications. The PCR reaction procedure was performed by $A B I$ 9700 PCR amplification (Applied Biosystems, Waltham, Massachusetts, USA) as follows. Predegeneration at $95^{\circ} \mathrm{C}$ for $2 \mathrm{~min}$, degeneration at $98^{\circ} \mathrm{C}$ for $10 \mathrm{~s}$, annealing at $62{ }^{\circ} \mathrm{C}$ for $30 \mathrm{~s}$, extension at $68{ }^{\circ} \mathrm{C}$ for $30 \mathrm{~s}$, with 27 cycles, extension at $68^{\circ} \mathrm{C}$ for $10 \mathrm{~min}$. PCR amplified products were detected and purified via $2 \%(\mathrm{~m} / \mathrm{v})$ Agarose gel electrophoresis. An Illumina Miseq PE300 sequencer (Illumina, San Diego, CA, USA) implemented the paired-end sequencing of PCR amplification.

The fastp software was adopted for the quality control of original sequencing sequence. The FLASH software was carried out for DNA fragment assembly, through which sequences of $<50 \mathrm{bp}$ and the reads with ambiguous bases, could be removed. The same operational taxonomic units (OTUs) were clustered by the sequences at the $97 \%$ identity threshold using UPARSE software. An $70 \%$ threshold was adopted to determine the taxonomic assignments of the OTUs through the Silva and Unite database. At OTUs level, the soil microbial community diversity, including the Chao 1 estimator and Shannon diversity, were calculated using MOTHUR software [26].

\section{Statistical analyses}

The independent-samples t-test was carried out to compare tea-leave chemical components, soil chemicophysical properties, and microbial community structure between ancient and modern tea plantations using SPSS 20.0 for Windows (Armonk, NY, USA). Principal coordinates analysis (PCoA) and non-metric multidimensional scaling (NMDS) were employed to evaluate the differences of soil microbial community composition according to Bray-Curtis distances between two types tea plantations using CANOCO software (Version 4.5). The interactions among tea-leaves chemical components, soil environmental factors and microbial community composition in tea plantation were confirmed through the bivariate correlation analysis of SPSS 20.0 for Windows (Armonk, NY, USA) and the redundancy analysis (RDA) of Origin 9.0 software (Hampton, Massachusetts, USA), respectively.

\section{Results And Discussion}


Except that the significant $(P<0.05)$ differences of chemical components were found in tea-leaves among five various geographic sites (Supplementary Table S1), the independent-samples t-test analysis indicated the definite differences of tea-leaves chemical components between modern and ancient tea plantations (Table 1), such as free amino acids and catechins, which revealed chemical components variations caused by geographical distribution and plantation type. In all sampling sites (Supplementary Table S1), tea-leaves collected from Bingdao possessed the highest levels of chemical components, including free amino acids, caffeine, gallic acid, GCG and ECG. Conversely, the tea-leaves from Baqishan had highest polyphenols/amino acids ratio value, and relatively higher contents of tea polyphenols, $\mathrm{C}$ and EGC, which was consistent with the report by Wang et al. (2018)[28], who discovered the differences of metabolic components in raw Pu-erh teas from various mountains.

Among 16 chemical indicators, 11 chemical indicators showed $(P<0.05)$, highly significant $(P<0.01)$ or extremely significant $(P<0.001)$ differences, including tea polyphenols, free amino acids, caffeine, gallic acid, EGC, GCG and EGCG, particularly polyphenols/amino acids ratio and non-ester/ ester catechins ratio with the extremely significant $(P<0.001)$ differences between modern and ancient tea plantations. Compared with modern tea plantations, ancient tea plantations were confirmed to possess higher contents of free amino acids, tea polyphenols, caffeine, gallic acid, EGC and total non-ester catechins, but had significantly $(P<0.05, P<0.01$ or $P<0.001)$ lower contents of GCG, EGCG and total ester catechins, which was consistent with the report by Cheng et al. (2018) [29] in EGCG content, while Ge et al. (2021) [13] discovered the catechins levels decreased after long-term plantation for about 1,000 years. The extremely significantly $(P<0.001)$ lower polyphenols/amino acids ratio and higher non-ester/ ester catechins ratio in fresh tea-leaves from ancient tea plantations indicated the significant difference in chemical compositions distribution of free amino acids, catechins and phenolic compounds between modern and ancient tea plantations. Generally, the average contents of free amino acids, gallic acid and EGC in tea-leaves from ancient tea plantations were 1.22, 6.22 and 1.67 times higher than that from modern tea plantations, which should be attributed to the physiological metabolisms of tea plant, such as amino acid metabolism, caffeine metabolism and phenolic compounds metabolism associated with the soil environment and ecological system of tea plantation. Due to the nitrogen metabolism in tea plant, several new flavoalkaloids were found in green tea collected from the ancient tea plantation [29].

\section{Soil chemicophysical properties and microbial quantity in various tea plantations}

Soil chemicophysical properties and microbial quantity varied significantly $(P<0.05)$ with five geographical sites and two types of tea plantations (Table 2 and Supplementary Table S2). The highest levels of SOM, AN and AP were observed in Bingdao site, while Jiulong site possessed the relatively lower soil fertility (Supplementary Table S2). Conversely, the soil pH (ranging from 4.18 to 4.77) kept relative stable among five geographical sites. About microbial quantity, there were no significant $(P \geq 0.05)$ differences between two types of tea plantations on the whole. However, fungi population was extremely significantly $(P<0.05)$ increased in ancient tea plantations $\left(11.25 \pm 3.54 \times 10^{3} \mathrm{CFU} \mathrm{g}^{-1}\right)$ compared with the modern tea plantations $\left(8.22 \pm 1.63 \times 10^{3} \mathrm{CFU} \mathrm{g}^{-1}\right)$, which indicated that the long-term plantation age or natural cultivation way improved fungi community quantity in ancient tea plantations. 
However, bacteria and actinomycetes kept relatively stable with no significant $(P \geq 0.05)$ variations in modern and ancient tea plantations.

Compared with the modern tea plantations, due to the micro-climate environment, low intensity production mode and less disturbance in cultivation management, the ancient tea plantations contained significantly $(P<0.05)$ higher soil fertility, including SOM, SOC, CEC, TN, TP, AN, AP and AK, but relative lower soil pH and TK. CEC (10.98-13.33 $\mathrm{cmol} \mathrm{kg}^{-1}$, range across all the soil samples), SOC (47.89-54.73 g $\left.\mathrm{kg}^{-1}\right)$, SOM (83.21-94.11 $\left.\mathrm{g} \mathrm{kg}^{-1}\right)$, TN $\left(1.50-1.87 \mathrm{~g} \mathrm{~kg}^{-1}\right)$, TP $\left(0.435-0.569 \mathrm{~g} \mathrm{~kg}^{-1}\right)$, TK $\left(4.11 \sim 6.31 \mathrm{~g} \mathrm{~kg}^{-1}\right)$, AN (113.99-143.78 $\left.\mathrm{mg} \mathrm{kg}^{-1}\right)$, AP (17.16-19.22 $\left.\mathrm{mg} \mathrm{kg}^{-1}\right)$ and AK (134.46-168.51 $\left.\mathrm{mg} \mathrm{kg}^{-1}\right)$ contents were accumulated in ancient tea plantations (Table 2). The soil in modern tea plantations was high acidification after 60-years plantation [30](Chen et al., 2020). Despite that the soil pH value (ranging from 4.38 to 4.55$)$ in ancient tea plantations was significantly $(P<0.05)$ lower than that in modern tea plantations, it was still optimum pH condition for the growth of tea trees [31]. The higher CEC, SOM and SOC in ancient tea plantations delayed soil acidification trend through relevant microbial metabolic activity [32].

In spite of a long-term plantation age over 100 years, the ancient tea plantations still kept relatively higher soil microbial metabolic activities, particularly fungi communities with an extremely significant $(P<0.001)$ increase, which should be attributed to the natural cultivation way of ancient tea plantations [33]. Generally, the ancient tea plantations were cultivated close to tall trees and sheltered by virgin forests that contributed to the accumulation of SOM and SOC due to the higher litter derived from tea plant and other plants [34]. The higher nitrogen $(N)$, phosphorus $(P)$ and potassium $(K)$ levels, particularly AN, AP and AK, ensure the sustainable development for the healthy growth of tea trees and the production of fresh tealeaves in the ancient tea plantations. Except the TK with a relatively higher loss that was translated into AK under the effect of related microbial communities, the natural cultivation way was conducive to a growth over 100 years in ancient tea plantations because of the higher soil fertility. The higher AN, AP and AK improved theanine and flavonoid biosynthesis activities in tea plant physiology through glutamine synthetase, phenylpropanoid, flavonoid pathways [35-36], which might contribute to the accumulations of free amino acids, gallic acid, caffeine and EGC in tea-leaves. Additionally, the photosynthetic capacity potentially impacted catechins biosynthesis (such as EGC) in tea plant [37], which might cause the significant differences of EGC, EGCG and GCG between modern and ancient tea plantations.

\section{Soil microbial community abundance and diversity in various tea plantations}

A total of 6978 OUTs belonging to 30 phyla, 125 classes, 293 orders, 455 families, 856 genera and 1818 species were obtained by analyzing the bacterial communities, while a total of 5525 OUTs belonging to 20 phyla, 68 classes, 170 families, 853 genera and 1408 species were obtained by analyzing the fungal communities in all soil samples through the Illumina high-throughput sequencing, respectively. The values of bacterial coverage estimators ranged from $96.94 \%$ to $97.70 \%$, and the values of fungal coverage estimators were all over $99.23 \%$ at $97 \%$ similarity cutoff, which suggested that the current 
numbers of the sequence reads were sufficient to reflect the soil bacterial and fungal community diversity (Supplementary Table S3).

Bacterial community abundance kept relatively stable among five geographic sites and two types of tea plantations ranging from 3375 OUTs to 4508 OUTs. Conversely, the fungal community abundance varied significantly $(P<0.05)$ with the geographic sites and tea plantation types. Generally, the ancient tea plantations involved a significant $(P<0.05)$ higher fungal community abundance. Therefore, plantation age enhanced the soil fungal community abundance in the natural cultivation way, while the bacterial community abundance kept stable with slight increases in the ancient tea plantations.

About a-diversity evaluated by Chao 1 and Shannon indices (Supplementary Table S3 and S4), soil bacterial abundance kept stable with no significant variations among five geographical sites and two types of tea plantations, except that the Chao 1 increased highly significantly in the ancient tea plantation (3215.36) of Jiulong site compared with the modern tea plantation (2754.08). However, Chao 1 and Shannon indices in soil fungal community diversity varied significantly $(P<0.05)$ with geographical sites and tea plantations type. Generally, a-diversity (i.e Chao 1 and Shannon indices) of fungal communities in ancient tea plantations were highly significantly $(P<0.01)$ or extremely significantly higher than that in modern tea plantations, respectively. Particularly, among five geographical sites, the ancient tea plantations located at Banuo, Dongguo and Jiulong sites showed significantly $(P<0.05)$ or highly significantly $(P<0.01)$ higher fungal community diversity than modern tea plantations. Compared with bacterial communities remaining stable along with the plantation age and cultivation ways, the fungal communities abundance and a-diversity significantly $(P<0.05)$ increased in the ancient tea plantations, which should be attributed to the long-term plantation age and natural cultivation way.

Previous studies confirmed that bacterial community diversity significantly $(P<0.05)$ decreased after longterm plantation over 23-years in modern tea plantations [19, 30]. However, despite the long-term plantation over 100 years, the bacterial community abundance and diversity kept stable in ancient tea plantations because of the natural cultivation way that maintained relatively higher soil fertility. The significantly $(P<0.05)$ higher fungal abundance and diversity in ancient tea plantations was consistent with the report by Wu et al. (2020)[38], which confirmed that the long-term plantation improve the fungal community in rhizosphere soil.

\section{Bacterial community composition in modern and ancient tea plantations}

Both PCoA (Fig. 2A) and NMDS (Supplementary Fig. S1) indicated the remarkable differences of bacterial community composition in soil aggregates between and ancient tea plantations. In the PCoA, the first two principal coordinates together represented $57.26 \%, 70.54 \%, 72.60 \%, 73.03 \%$ and $77.31 \%$ of the variations by PC1 and PC2 in soil bacterial community composition from two types of tea plantations located at Bingdao, Baqishan, Banuo, Dongguo and Jiulong, respectively, which demonstrated that the bacterial community from same type of tea plantation in five various geographical sites was almost grounded together, while the bacterial community from the ancient tea plantation in the same geographical site were well separated from each other. Additionally, NMDS analysis revealed that the bacterial community 
composition between modern and ancient tea plantations in the same geographical site was significantly $(P<0.05)$ distinguished.

At the phylum level in soil bacterial community composition (Fig. 2B), Chloroflexi (24.94\%), Proteobacteria (22.39\%), Acidobacteriota (17.00\%) and Actinobacteriota (13.15\%) were regarded as the dominant groups in ancient tea plantations, which kept relatively stable in modern tea plantation with no significant $(P \geq 0.05)$ variations. Compared to the modern tea plantations, Proteobacteria and Actinobacteriota showed slight downward trend, while Chloroflexi and Acidobacteriota showed slight rising tendency in ancient tea plantations. Particularly, due to the long-term plantation and natural cultivation way, the relative abundances of Nitrospirota and Methylomirabilota significantly $(P<0.05)$ or highly significantly $(P<0.01)$ increased, but the relative abundances of Bacteroidota, Patescibacteria and Bdellovibrionota significantly $(P<0.05)$ or extremely significantly $(P<0.001)$ decreased in the ancient tea plantations (Supplementary Table S5).

At the genus level, c_AD3 (12.01\%), f_Xanthobacteraceae (5.62\%), o_Acidobacteriales (4.38\%), o_Subgroup_2 (3.04\%), o_Vicinamibacterales (2.57\%), Bradyrhizobium (2.22\%), f_Gemmataceae (2.07\%), HSB_OF53-F07 (2.05\%), o_IMCC26256 (2.03\%) and C_TK10 (2.01\%) were the dominate genera in bacterial community of ancient tea plantations. Particularly, $\mathrm{f} \_$Xanthobacteraceae, $\mathrm{c} \_$TK10 and HSB_OF53-F07 significantly $(P<0.05)$ increased in the ancient tea plantations. Additionally, the stabilization of o_Acidobacteriales and f_Acidobacteriaceae_Subgroup_1 contributed to the soil pH in ancient tea plantations. Among the top 50 bacterial genera in relative abundance (Supplementary Table S6), compared with the modern tea plantations, 5 genera (i.e. f__Xanthobacteraceae, C_TK10, HSB_OF53-F07, O_Rokubacteriales and Nitrospira) significantly $(P<0.05)$ or highly significantly $(P<0.01)$ increased, while 6 genera (i.e. Sphingomonas, Burkholderia-Caballeronia-Paraburkholderia, Mycobacterium, Haliangium, Gemmatimonas and Kitasatospora) significantly $(P<0.05)$ decreased in the ancient tea plantations. Particularly, as the main nitrifying bacteria, the improvement of Nitrospirota phylum and Nitrospira genus in relative abundance significantly $(P<0.05)$ enhanced the level of AN and soil fertility of ancient tea plantations. Additionally, HSB_OF53-F07 and Acidothermus genera improved the soil carbon metabolism in ancient tea plantations.

The dominant phyla in bacterial community composition of tea plantations were consistent with the studies of Wang et al. (2019)[19] and Zheng et al. (2020)[39], while Chen et al. (2020) [30] discovered that Proteobacteria, Acidobacteriota, Actinobacteriota and Firmicutes were dominate phyla in soil of tea plantations. Although Actinobacteriota increased toward lower soil pH often [40], it kept stable in relative abundance in ancient tea plantations. As the main soil probiotics, the slight improvement of Bacillus genus enhanced the soil fertility and conduced to the sustainable development of ancient tea plantations.

\section{Fungal community composition in modern and ancient tea plantations}

Similar to bacterial community composition, fungal community composition varied significantly $(P<0.05)$ with geographical site and tea plantation types through PCoA (Fig. 3A) and NMDS analysis (Supplementary Fig. S2). In the PCoA, the first two principal coordinates together represented $55.90 \%$, 
$48.10 \%, 65.79 \%, 57.62 \%$ and $45.75 \%$ of the variations by PC1 and PC2 in soil fungal community composition between modern and ancient tea plantations located at Bingdao, Baqishan, Banuo, Dongguo and Jiulong, respectively, which indicated the remarkable differences in soil fungal community composition between modern and ancient tea plantations. The results of PCoA were consistent with NMDS analysis (Supplementary Fig. S2), demonstrated the extremely significantly $(P<0.001)$ distinction in fungal community composition between two types of tea plantations in the same geographical site.

At the phylum level in soil fungal community composition (Fig. 3B), Ascomycota (65.75\%), Basidiomycota (19.02\%), Mortierellomycota (5.08\%), Rozellomycota (1.61\%), Glomeromycota (0.39\%) and Unclassified-k-Fungi (7.57\%) were the dominant groups in ancient tea plantations. Except for Jiulong, the relative abundance of Ascomycota in the ancient tea plantations of other four sites was significantly $(P<0.05)$ higher than that in modern tea plantations, which occupied from $51.50 \%$ to $70.28 \%$. The soil fungal community composition were significant $(P<0.05)$ differences across five sampling sites. For example, the relative abundance of Rozellomycota in modern tea plantation of Dongguo site reached $20.77 \%$, but its relative abundance in other soil aggregates were relatively low. Compared to modern tea plantations, Unclassified-k-Fungi and Rozellomycota significantly $(P<0.05)$ decreased, while Ascomycota and Mortierellomycota significantly $(P<0.05)$ increased in the ancient tea plantations (Supplementary Table S7).

At the genus level, Saitozyma (11.17\%), p_Ascomycota (11.15\%), Unclassified_k_Fungi (7.57\%), Mortierella (5.02\%), Chaetomium (4.32\%), Penicillium (3.33\%), o_Chaetothyriales (3.31\%), C_Eurotiomycetes (2.89\%), f_Didymellaceae (2.30\%), f_Clavariaceae (2.19\%) and Fusarium (1.82\%) were identified as the dominate genera in ancient tea plantations (Supplementary Table S8). Compared to the modern tea plantations, the relative abundances of Saitozyma, Metarhizium, Mortierella, f_Clavariaceae, Pseudogymnoascus, o__Helotiales, Leohumicola, Tolypocladium and Trichoglossum significantly $(P<0.05)$, highly significantly $(P<0.01)$ or extremely significantly $(P<0.001)$ increased in the ancient tea plantations. Additionally, 3 genera including Unclassified_k_Fungi, p_Rozellomycota and Phialocephala significantly $(P<0.05)$ decreased in ancient tea plantations.

The dominant phyla in fungal community composition were consistent with the report by Wu et al. (2020) [38]. The abundances of Ascomycota and Mortierellomycota phyla, as well as 9 genera (i.e. Saitozyma, Metarhizium, Mortierella, f_Clavariaceae, Pseudogymnoascus, o_Helotiales, Leohumicola, Tolypocladium and Trichoglossum) significantly $(P<0.05)$ increased with the plantation age in natural cultivation way, which should be attributed to the higher fertility in the ancient tea plantations. The entomopathogenic fungi stay at relatively lower level in ancient tea plantation. For instance, the abundance of Fusarium genus showed a slight decrease from $2.86 \%$ to $1.82 \%$ in the ancient tea plantation.

\section{Potential impact of soil environmental factors in tea plantations}

The bivariate correlation analysis was carried out to explore the relevance among soil environmental factors including soil fertility and microbial population, and tea-leaves quality components in tea 
plantations through the relevant Spearman correlation coefficient (Supplementary Table S9 and Table S10). The soil TN, TP, AN, and AP levels might improved the contents of free amino acids, gallic acid and EGC in tea-leaves with the significant $(P<0.05)$ or highly significant $(P<0.01)$ positive correlations ( $r$-value ranging from 0.355 to 0.624$)$, but decreased EGCG content with the highly significant $(P<0.01)$ negative correlations ( $r$-value $=-0.451,-0.471$ and -0.611 , respectively). Additionally, TK and AK impacted potentially the levels of phenolic components including gallic acid, C, EC, EGC, ECG, GCG and EGCG, due to the significant $(P<0.05)$ or highly significant $(P<0.01)$ negative correlations (Supplementary Table S9). These results indicated that soil environmental factors, such as CEC, SOC, SOM, nitrogen (N), phosphorus $(\mathrm{P})$ and potassium $(\mathrm{K})$, showed significant $(P<0.05)$ associations with tea-leaves quality components, particularly free amino acids and phenolic components. Generally, SOC, SOM, N and P contributed to the accumulations of free amino acids, gallic acid and EGC, while the $\mathrm{K}$ might impacted the biosynthesis of phenolic compounds, including gallic acid, C, EC, EGC, ECG, GCG and EGCG, which generated the chemical differences in tea-leaves between modern and ancient tea plantations.

The soil $\mathrm{pH}$ was main environmental factor impacting the microbial abundance and diversity. In the modern tea plantations, the bacterial abundance and diversity decreased significantly $(P<0.05)$ after the long-term plantation due to the continuous acidification in the soil $[19,28]$. However, despite the reduction of bacterial abundance with the lower soil pH, the relatively lower soil pH improved the abundances of actinomycetes and fungi in ancient tea plantations. Additionally, the microbial diversity including bacterial and fungal diversity was not sensitive to soil pH (Supplementary Table S10). Differed form soil $\mathrm{pH}$, the soil fertility, such as SOC, SOM and TP, might improve fungi abundance determined by dilution coating method. Additionally, the soil $\mathrm{P}$ level improved the abundance and diversity of fungi community, and actinomycetes richness with the significantly $(P<0.05)$ or highly significantly $(P<0.01)$ positive correlations, while AK level showed highly significantly positive correlation to bacterial community abundance. The $\mathrm{N}$ level in soil had limited influence on soil microbial community abundance and diversity with no significant $(P \geq 0.05)$ correlations to bacterial and fungal communities.

\section{Interactions between soil physical properties and microbial community composition}

The Spearman correlation coefficient determined by the bivariate correlation analysis corresponded highly to the RDA. In this study, the top 22 bacterial phyla (Fig. 4A) and 12 fungal phyla in microbial communities were carried out as the response variables through the RDA to elaborate the impact of soil environmental factors on microbial community composition in tea plantations, respectively. The RDA model accounted for $93.56 \%$ and $86.87 \%$ of the total variation of soil bacterial and fungal community composition in tea plantations, respectively. AK, AN, TP, CEC and TK were main environmental factors affecting bacterial community composition in turn (Fig. 4A). CEC, TK and soil pH showed significantly positive correlations with the relative abundances of Proteobacteria and Acidobacteriota, while the positive correlations of the relative abundances of Actinobacteria and Chloroflexi to AK, AN, and AP were found in the bacterial community composition (Fig. 4A). Therefore, CEC contributed to the improvement of $\mathrm{f}$ __Xanthobacteraceae, while TK and soil pH impacted Sphingomonas and Burkholderia-CaballeroniaParaburkholderia genera in ancient tea plantations. Additionally, the soil AN, AP and AK impacted 
significantly $(P<0.05)$ the abundances of C__TK10, HSB_OF53-F07, Mycobacterium and Kitasatospora in tea plantations.

However, for the fungal community composition in tea plantations, TP, AN, AP, pH and AK were main influence factors. The abundance of Ascomycota in fungal community composition showed significant positive correlations to soil chemical properties, such as AN, AP, AK, SOC, SOM and TP, but with negative correlation to soil $\mathrm{pH}$, which indicated that the soil chemical properties improved the abundance of Ascomycota including Metarhizium, Pseudogymnoascus, o_Helotiales, Leohumicola, Tolypocladium and Trichoglossum genera toward to the lower $\mathrm{pH}$ in the ancient tea plantations. Soil pH and TN showed definitely positive corrections to the abundances of Unclassified_k_Fungi, Basidiomycota and Rozellomycota, which revealed that these fungal phyla were sensitive to the soil $\mathrm{pH}$ with significant $(P<0.05)$ decreases in ancient tea plantations (Fig. 4B). Generally, the significant $(P<0.05)$ increases of Ascomycota phylum as well as Metarhizium, Pseudogymnoascus, o_Helotiales, Leohumicola, Tolypocladium and Trichoglossum genera in the abundances caused by the higher soil fertility and lower $\mathrm{pH}$ value contributed to the stabilization of soil microbial diversity and abundance in ancient tea plantations.

\section{Conclusions}

This study confirmed that tea-leaves chemical components and soil chemicophysical properties, as well as microbial community abundance and diversity in soil varied significantly $(P<0.05)$ with the geographical sites and tea plantation type through HPLC and high-throughput sequencing analysis. Compared with the modern tea plantations, ancient tea plantations were observed to possess significantly $(P<0.05)$ higher free amino acids, tea polyphenols, caffeine, gallic acid and EGC contents, but significant lower GCG and EGCG contents in tea-leaves. Additionally, the ancient tea plantations contained significantly $(P<0.05)$ higher soil fertility, including CEC, SOC, SOM, TN, TP, AN, AP and AK, but significantly lower TK and $\mathrm{pH}$ value, which should be attributed to the excellent ecological environment, natural cultivation way and complete community structure of ancient tea plantations. Combined with the dilution coating method, high-throughput sequencing analysis revealed that the abundances and diversity of bacteria and actinomycetes kept relatively stable, while fungal community abundance and diversity significantly $(P<0.05)$ increased in soil of ancient tea plantations.

PCOA and NMDS analysis indicated the significant $(P<0.05)$ and highly significant $(P<0.05)$ differences in bacterial and fungal community compositions between modern and ancient tea plantations, respectively. The long-term plantation in natural cultivation way significantly $(P<0.05)$ improved the abundances of Nitrospirota and Methylomirabilota phyla, as well as 5 genera (i.e. f__Xanthobacteraceae, C__TK10, HSB_OF53-F07, o_Rokubacteriales and Nitrospira) in bacterial community composition, and the abundances of Ascomycota and Mortierellomycota phyla as well as 9 genera (i.e. Saitozyma, Metarhizium, Mortierella, f__Clavariaceae, Pseudogymnoascus, o__Helotiales, Leohumicola, Tolypocladium and Trichoglossum) in fungal community composition of ancient tea plantations. The bivariate correlation analysis and RDA confirmed that SOC, SOM, N and P contributed to the 
accumulations of free amino acids, gallic acid and EGC in ancient tea plantations. Additionally, AK, AN, TP, CEC and TK impacted the abundances of Proteobacteria, Acidobacteriota, Actinobacteria and Chloroflexi in bacterial community composition, while the higher soil fertility and lower $\mathrm{pH}$ value significantly $(P<0.05)$ enhanced the abundance of Ascomycota including Metarhizium, Pseudogymnoascus, o_Helotiales, Leohumicola, Tolypocladium and Trichoglossum genera in fungal community composition of ancient tea plantations.

By contrast with the modern tea plantations, this study revealed the characteristics of tea-leaves chemical components, soil chemicophysical properties and microbial community structure in the ancient tea plantations. The significantly $(P<0.05)$ higher soil fertility promised the stabilization of bacterial abundance and diversity, and improved the abundance and diversity of fungal community in ancient tea plantations. Generally, this study elaborated the sustainable development of ancient tea plantations in soil ecosystem.

\section{Declarations}

\section{CRediT authorship contribution statement}

Guangrong Yang, Dapeng Zhou, Jin Xie and Cunqiang Ma: Conceived and designed the study, analysed data and drafted the manuscript. Dapeng Zhou, Renyuan Wan and Conglian Wang: performed the experiments. Yongmei Li: Revised the manuscript.

\section{Declaration of competing interest}

The authors declare that they have no known competing financial interests or personal relationships that could have appeared to influence the work reported in this paper.

\section{Acknowledgements}

This research was financially supported by National Natural Science Foundation of China (Grant No. 31660225) and Doctoral Research Foundation of Yunnan Agricultural University (A2002338). In addition, we also thank Shanghai Biotree Biotech Co. Ltd for the assistance in genomic analysis.

\section{Ethics approval}

The study did not involve any human or animal testing.

\section{References}

1. Li Q, Huang J, Li Y, Zhang Y, Luo Y, Chen Y, et al. Fungal community succession and major components change during manufacturing process of Fu brick tea. Scientific Reports, 2017;7(1):6947. 
2. Ma C, Li X, Zheng C, Zhou B, Xu C, Xia T. Comparison of characteristic components in tea-leaves fermented by Aspergillus pallidofulvus PT-3, Aspergillus sesamicola PT-4 and Penicillium manginii PT-5 using LC-MS metabolomics and HPLC analysis. Food Chemistry, 2021;350:129228.

3. Huang F, Zheng X, Ma X, Jiang R, Zhou W, Zhou S, et al. Theabrownin from Pu-erh tea attenuates hypercholesterolemia via modulation of gut microbiota and bile acid metabolism. Nature Communications, 2019;10:4971.

4. Wei C, Yang H, Wang S, Zhao J, Liu C, Gao L, et al. Draft genome sequence of Camellia sinensis var. sinensis provides insights into the evolution of the tea genome and tea quality. PNAS, 2018;115(18):E4151-E4158.

5. Jin JQ, Yao MZ, Ma CL, Ma JQ, Chen L. Association mapping of caffeine content with TCS1 in tea plant and itsrelated specie. Plant Physiology and Biochemistry, 2016;100:18-26.

6. Niu S, Song Q, Koiwa H, Qiao D, Zhao D, Chen Z, et al. Genetic diversity, linkage disequilibrium, and population structure analysis of the tea plant (Camellia sinensis) from an origin center, Guizhou plateau, using genome-wide SNPs developed by genotyping-by-sequencing. BMC Plant Biology, 2019;19:328.

7. Zi H, Jiang $Y$, Cheng $X$, Li W, Huang $X$. Change of rhizospheric bacterial community of the ancient wild tea along elevational gradients in Ailao mountain, China. Scientific Reports, 2020;10(1):9203.

8. Zhang W, Liu C, Yang R, Zheng T, Zhao M, Ma L, et al. Comparison of volatile profiles and bioactive components of sun-dried Pu-erh tea leaves from ancient tea plants on Bulang Mountain measured by GC-MS and HPLC. Journal of Zhejiang University-Science B, 2019;20(7):563-575.

9. Xia EH, Zhang HB, Sheng J, Li K, Zhang QJ, Kim C,.et al. The tea tree genome provides insights into tea flavor and independent evolution of caffeine biosynthesis. Molecular Plant, 2017;10(6):866-877.

10. Zhou B, Wang Z, Yin P, Ma B, Ma C, Xu C, et al. Impact of prolonged withering on phenolic compounds and antioxidant capability in white tea using LC-MS-based metabolomics and HPLC analysis: Comparison with green tea. Food Chemistry, 2022;368:130855.

11. Zhou B, Ma B, Ma C, Xu C, Wang J, Wang Z, et al. Classification of Pu-erh ripened teas and their differences in chemical constituents and antioxidant capacity. LWT-Food Science and Technology, 2022;153:112370.

12. Xia X, Wang X, Wang H, Lin Z, Shao K, Xu J, et al. Ameliorative effect of white tea from 50-year-old tree of Camellia sinensis $\mathrm{L}$. (Theaceae) on kidney damage in diabetic mice via SIRT1/AMPK pathway. Journal of Ethnopharmacology, 2021;272:113919.

13. Ge Y, Li N, Fu Y, Yu X, Xiao Y, Tang Z,.et al. Deciphering superior quality of Pu-erh tea from thousands of years' old trees based on the chemical profile. Food Chemistry, 2021;358:129602.

14. Xu S, Wang JJ, Wei Y, Deng WW, Wan X, Bao GH, et al. Metabolomics based on UHPLC-Orbitrap-MS and global natural product social molecular networking reveals effects of time scale and environment of storage on the metabolites and taste quality of raw pu-erh tea. Journal of Agricultural and Food Chemistry, 2019;67(43):12084-12093. 
15. Zhang J, Yang R, Li YC, Peng Y, Wen X, Ni X. Distribution, accumulation, and potential risks of heavy metals in soil and tea leaves from geologically different plantations. Ecotoxicology and Environmental Safety, 2020;195:110475.

16. Li W, Zheng Z, Li T, Zhang X, Wang Y, Yu H, et al. Effect of tea plantation age on the distribution of soil organic carbon fractions within water-stable aggregates in the hilly region of Western Sichuan, China. Catena, 2015;133:198-205.

17. Ludwig M, Achtenhagen J, Miltner A, Eckhardt KU, Leinweber P, Emmerling C, et al. Microbial contribution to SOM quantity and quality in density fractions of temperate arable soils. Soil Biology \& Biochemistry,2015;81:311-322.

18. Sun L, Xun W, Huang T, Zhang G, Gao J, Ran W, et al. Alteration of the soil bacterial community during parent material maturation driven by different fertilization treatments. Soil Biology \& Biochemistry,2016;96:207-215.

19. Wang S, Li T, Zheng Z, Chen HY. Soil aggregate-associated bacterial metabolic activity and community structure in different aged tea plantations. Science of the Total Environment, 2019;654:1023-1032.

20. Zhu R, Zheng Z, Li T, He S, Zhang X, Wang Y, et al. Effect of tea plantation age on the distribution of glomalin-related soil protein in soil water-stable aggregates in southwestern China. Environmental Science and Pollution Research, 2019;26:1973-1982.

21. Liu Y, Zhao G, Li X, Shen Q, Wu J, Zhuang J, et al. Comparative analysis of phenolic compound metabolism among tea plants in the section Thea of the genus Camellia. Food Research International, 2020;135:109276.

22. Zhou B, Ma C, Wu T, Xu C, Wang J, Xia T. (2020). Classification of raw Pu-erh teas with different storage time based on characteristic compounds and effect of storage environment. LWT-Food Science and Technology, 2020;133:109914.

23. Yang G, Wang X, Xie J, Lv C, Li C. Analysis of the Relationship between soil nutrients and tea main quality components of ancient tea arboretum and modern tea garden in Yunnan Province. Journal of Tea Science, 2015;35(6):574-582.(in Chinese)

24. Zhou B, Ma C, Wang H, Xia T. Biodegradation of caffeine by whole cells of tea-derived fungi Aspergillus sydowii, Aspergillus niger and optimization for caffeine degradation. BMC Microbiology, 2018;18: 53.

25. Tang, H., Xiao, X., Li, C., Wang, K., Guo, L., Cheng, K., Sun, G., Pan, X. (2018). Impact of long-term fertilization practices on the soil aggregation and humic substances under double-cropped rice fields. Environmental Science and Pollution Research, 25, 11034-11044.

26. Wu W, Dong C, Wu J, Liu X, Wu Y, Chen X, Yu S. Ecological effects of soil properties and metal concentrations on the composition and diversity of microbial communities associated with land use patterns in an electronic waste recycling region. Science of the Total Environment, 2017;601:57-65.

27. Zheng N, Yu Y, Shi W, Yao H. Biochar suppresses $\mathrm{N} 20$ emissions and alters microbial communities in an acidic tea soil. Environmental Science and Pollution Research, 2019;26:35978-35987. 
28. Wang T, Li X, Yang H, Wang F, Kong J, Qiu D, et al. Mass spectrometry-based metabolomics and chemometric analysis of Pu-erh teas of various origins. Food Chemistry, 2018;268:271-278.

29. Cheng J, Wu FH, Wang P, Ke JP, Wan XC, Qiu MH, et al. Flavoalkaloids with pyrrolidinone ring from Chinese Ancient cultivated tea Xi-Gui. Journal of Agricultural and Food Chemistry,2018;66(30):79487957

30. Chen P, Liu Y, Mo C, Jiang Z, Lin J. Microbial mechanism of biochar addition on nitrogen leaching and retention in tea soils from different plantation ages. Science of the Total Environment, 2020;757:143817.

31. Koga K, Suehlro Y, Matsuoka ST, Takahashi K.(2003). Evaluation of growth activity of microbes in tea field soil using microbial calorimetry. $<$ bi $>$ Journal of Bioscience and Bioengineering $</ \mathrm{bi}>$, 2003:95(5):429-434.

32. Lin W, Lin M, Zhou H, Wu H, Li Z, Lin W. The effects of chemical and organic fertilizer usage on rhizosphere soil in tea orchards. PLoS One, 2019;14(5):e0217018.

33. Hung P.Y. Tea forest in the making: Tea production and the ambiguity of modernity on China's southwest frontier. Geoforum, 2013;47:178-188.

34. Pramanik P, Phukan M. Assimilating atmospheric carbon dioxide in tea gardens of northeast India. Journal of Environmental Management, 2020;256:109912.

35. Fu X, Liao Y, Cheng S, Xu X, Grierson D, Yang Z. Nonaqueous fractionation and overexpression of fluorescent-tagged enzymes reveals the subcellular sites of L-theanine biosynthesis in tea. Plant Biotechnology Journal, 2021;19(1):98-108.

36. Wang Y, Cheng X, Yang T, Su Y, Lin S, Zhang S, et al. Nitrogen-regulated theanine and flavonoid biosynthesis in tea plant roots: Protein-level regulation revealed by multiomics analyses. Journal of Agricultural and Food Chemistry, 2021;39(34):10002-10016.

37. Xiang P, Zhu Q, Tukhvatshin M, Cheng B, Tan M, Liu J, et al. Light control of catechin accumulation is mediated by photosynthetic capacity in tea plant (Camellia sinensis). BMC Plant Biology, 2021;21:478.

38. Wu Z, Su Q, Cui Y, He H, Wang J, Zhang Y, et al.Temporal and spatial pattern of endophytic fungi diversity of Camellia sinensis (cv. Shu Cha Zao). BMC Microbiology, 2020;20:270.

39. Zheng N, Yu Y, Wang J, Chapman SJ, Yao H, Zhang Y. The conversion of subtropical forest to tea plantation changes the fungal community and the contribution of fungi to $\mathrm{N} 2 \mathrm{O}$ production. Environmental Pollution, 2020;265:115106.

40. Wang H, Zeng Y, Guo C, Bao,Y, Lu G, Reinfelder JR, et al. (2018). Bacterial, archaeal, and fungal community responses to acid mine drainage-laden pollution in a rice paddy soil ecosystem. Science of the Total Environment, 2018;616:107-116.

\section{Tables}

Table 1 Chemical indicator of tea-leaves in modern and ancient tea plantations, respectively. 


\begin{tabular}{|c|c|c|c|c|}
\hline \multirow[t]{2}{*}{ Item } & \multicolumn{2}{|c|}{ Modern tea gardens } & \multicolumn{2}{|c|}{ Ancient tea gardens } \\
\hline & Mean $(n=5 * 5)$ & Rang & Mean $(n=5 * 5)$ & Rang \\
\hline Water extraction (\%) & $49.74 \pm 2.65$ & $48.65 \sim 50.84$ & $50.82 \pm 2.09$ & $49.96 \sim 51.69$ \\
\hline Free amino acids (\%) & $2.81 \pm 0.36$ & $2.65 \sim 2.96$ & $3.43 \pm 0.30 * * *$ & $3.31 \sim 3.55$ \\
\hline Tea polyphenols (\%) & $29.47 \pm 1.44$ & $28.87 \sim 30.06$ & $30.83 \pm 1.77 * *$ & $30.10 \sim 31.56$ \\
\hline Polyphenols/Amino acids ratio & $10.74 \pm 2.03$ & $9.90 \sim 11.58$ & $9.08 \pm 1.23^{* * *}$ & $8.57 \sim 9.59$ \\
\hline Caffeine $\left(\mathrm{mg} \mathrm{g}^{-1}\right)$ & $40.21 \pm 3.93$ & $38.59 \sim 41.83$ & $42.78 \pm 3.33^{*}$ & $41.40 \sim 44.15$ \\
\hline Gallic acid (mg g $\left.{ }^{-1}\right)$ & $0.09 \pm 0.18$ & $0.01 \sim 0.16$ & $0.56 \pm 0.11 * * *$ & $0.51 \sim 0.60$ \\
\hline $\mathrm{C}\left(\mathrm{mg} \mathrm{g}^{-1}\right)$ & $15.74 \pm 4.94$ & $13.70 \sim 17.78$ & $13.85 \pm 5.45$ & $11.60 \sim 16.11$ \\
\hline $\mathrm{EC}\left(\mathrm{mg} \mathrm{g}^{-1}\right)$ & $28.34 \pm 7.60$ & $25.20 \sim 31.47$ & $30.10 \pm 10.33$ & $25.84 \sim 34.36$ \\
\hline $\operatorname{EGC}\left(\mathrm{mg} \mathrm{g}^{-1}\right)$ & $18.20 \pm 5.45$ & $15.95 \sim 20.44$ & $30.47 \pm 3.52^{* * *}$ & $29.02 \sim 31.93$ \\
\hline $\mathrm{ECG}\left(\mathrm{mg} \mathrm{g}^{-1}\right)$ & $85.66 \pm 19.44$ & $77.64 \sim 93.69$ & $75.04 \pm 20.66$ & $66.51 \sim 83.57$ \\
\hline GCG $\left(\mathrm{mg} \mathrm{q}^{-1}\right)$ & $5.45 \pm 1.50$ & $4.83 \sim 6.07$ & $4.35 \pm 1.78 *$ & $3.61 \sim 5.08$ \\
\hline $\operatorname{EGCG}\left(\mathrm{mg} \mathrm{g}^{-1}\right)$ & $53.40 \pm 6.28$ & $50.81 \sim 55.99$ & $44.92 \pm 4.70^{* * *}$ & $42.98 \sim 46.86$ \\
\hline Total catechins ( $\mathrm{mg} \mathrm{g}^{-1}$ ) & $200.79 \pm 35.87$ & $191.98 \sim 221.60$ & $198.74 \pm 37.51$ & $183.25 \sim 214.22$ \\
\hline Non-ester catechins $\left(\mathrm{mg} \mathrm{g}^{-1}\right)$ & $62.28 \pm 16.70$ & $55.38 \sim 69.17$ & $74.43 \pm 15.51 *$ & $68.02 \sim 80.83$ \\
\hline Ester catechins $\left(\mathrm{mg} \mathrm{g}^{-1}\right)$ & $144.51 \pm 22.38$ & $135.28 \sim 153.75$ & $124.31 \pm 22.91 * *$ & $114.85 \sim 133.76$ \\
\hline Non-ester/ Ester catechins ratio & $0.43 \pm 0.09$ & $0.39 \sim 0.46$ & $0.60 \pm 0.05^{* * *}$ & $0.57 \sim 0.62$ \\
\hline
\end{tabular}

Note: Polyphenols/Amino acids ratio $=$ Tea polyphenols content/ free amino acids content; Total catechins were the summation of C, EC, EGC, ECG, GCG and EGCG contents; Non-ester catechins were the summation of C, EC and EGC contents; Ester catechins were the summation of ECG, GCG and EGCG contents; Non-ester/ Ester catechins ratio $=$ Non-ester catechins content / Ester catechins content.

And all data were present by mean value \pm standard deviation. The range was the mean value at $95 \%$ confidence coefficient. Significance levels: ${ }^{*} P<0.05 ;{ }^{* *} P<0.01$ and ${ }^{* * *} P<0.001$ determined by the independent-samples t-test using SPSS 20.0 for Windows.

Table 2 Soil environmental factors and microbial population in modern and ancient tea plantations, respectively.

\begin{tabular}{|c|c|c|c|c|c|}
\hline \multirow[t]{2}{*}{ Item } & & \multicolumn{2}{|c|}{ Modern tea plantations } & \multicolumn{2}{|c|}{ Ancient tea plantations } \\
\hline & & Mean $(n=5 * 5)$ & Rang & Mean $(n=5 * 5)$ & Rang \\
\hline \multirow{10}{*}{$\begin{array}{l}\text { Soil physical and } \\
\text { chemical properties }\end{array}$} & pH value & $4.59 \pm 0.13$ & $4.53 \sim 4.64$ & $4.47 \pm 0.21 *$ & $4.38 \sim 4.55$ \\
\hline & CEC $\left(\mathrm{cmol} \mathrm{kg}{ }^{-1}\right)$ & $9.13 \pm 3.34$ & $7.75 \sim 10.51$ & $12.15 \pm 2.84^{* *}$ & $10.98 \sim 13.33$ \\
\hline & SOC $\left(\mathrm{g} \mathrm{kg}^{-1}\right)$ & $38.67 \pm 12.56$ & $33.49 \sim 43.86$ & $51.31 \pm 8.29^{* * *}$ & $47.89 \sim 54.73$ \\
\hline & $\operatorname{SOM}\left(\mathrm{g} \mathrm{kg}^{-1}\right)$ & $65.93 \pm 21.88$ & $56.90 \sim 74.96$ & $88.66 \pm 13.20^{* * *}$ & $83.21 \sim 94.11$ \\
\hline & $\mathrm{TN}\left(\mathrm{g} \mathrm{kg}^{-1}\right)$ & $1.19 \pm 0.49$ & $0.98 \sim 1.39$ & $1.68 \pm 0.45^{* *}$ & $1.50 \sim 1.87$ \\
\hline & $\mathrm{TP}\left(\mathrm{g} \mathrm{kg}^{-1}\right)$ & $0.257 \pm 0.122$ & $0.206 \sim 0.307$ & $0.502 \pm 0.162^{* * *}$ & $0.435 \sim 0.569$ \\
\hline & $\mathrm{TK}\left(\mathrm{g} \mathrm{kg}^{-1}\right)$ & $6.59 \pm 1.19$ & $6.10 \sim 7.08$ & $5.21 \pm 2.67 *$ & $4.11 \sim 6.31$ \\
\hline & $\mathrm{AN}\left(\mathrm{mg} \mathrm{kg}^{-1}\right)$ & $100.55 \pm 26.04$ & $89.80 \sim 111.29$ & $128.88 \pm 36.09 * *$ & $113.99 \sim 143.78$ \\
\hline & $\mathrm{AP}\left(\mathrm{mg} \mathrm{kg}^{-1}\right)$ & $10.64 \pm 3.41$ & $9.23 \sim 12.05$ & $18.19 \pm 2.49 * * *$ & $17.16 \sim 19.22$ \\
\hline & $\mathrm{AK}\left(\mathrm{mg} \mathrm{kg}^{-1}\right)$ & $132.76 \pm 14.57$ & $126.74 \sim 138.78$ & $151.49 \pm 41.24 *$ & $134.46 \sim 168.51$ \\
\hline \multirow[t]{3}{*}{$\begin{array}{l}\text { Soil } \\
\text { population }\end{array}$} & $\begin{array}{l}\text { Bacteria } \quad\left(\times 10^{6} \mathrm{CFU}\right. \\
\left.\mathrm{g}^{-1}\right)\end{array}$ & $20.88 \pm 7.72$ & $17.70 \sim 24.07$ & $20.22 \pm 3.86$ & $18.63 \sim 21.81$ \\
\hline & $\begin{array}{l}\text { Fungi }\left(\times 10^{3} \mathrm{CFU} \mathrm{g}^{-}\right. \\
\left.{ }^{-}\right)\end{array}$ & $8.22 \pm 1.63$ & $7.54 \sim 8.89$ & $11.25 \pm 3.54^{* * *}$ & $9.79 \sim 12.71$ \\
\hline & $\begin{array}{l}\text { Actinomycetes } \\
\left(\times 10^{7} \mathrm{CFU} \mathrm{g}^{-1}\right)\end{array}$ & $9.77 \pm 7.15$ & $6.82 \sim 12.72$ & $10.18 \pm 4.75$ & $8.22 \sim 12.15$ \\
\hline
\end{tabular}

CEC: Cation exchange capacity; SOC: Soil organic carbon; SOM: Soil organic matter; TN: Total nitrogen; TP: Total phosphorus; TK: Total potassium; AN: Alkali-hydrolyzable nitrogen; AP: Available phosphorus; AK: Available potassium.

All data were present by mean value \pm SD. The range was the mean value at $95 \%$ confidence coefficient. Significance levels: * $P<0.05$; ** $P<0.01$ and *** $P<0.001$ determined by the independent-samples t-test using SPSS 20.0 for Windows. 
Table S1 Chemical components in tea-leaves of modern and ancient tea plantations in five sampling sites including Bingdao, Baqishan, Banuo, Dongguo and Jiulong, respectively.

Table S2 Soil nutrition and microbial population of modern and ancient tea plantations in five sampling sites including Bingdao, Baqishan, Banuo, Dongguo and Jiulong, respectively.

Table S3 Soil bacterial and fungal community diversity (Chao 1, Shannon and Coverage) of modern and ancient tea plantations in the five sampling sites, respectively.

Table S4 Bacterial and fungal community diversity (Chao 1 and Shannon) differences in soil between modern and ancient tea plantations.

Table S5 Differences of the relative abundance of the top 22 bacterial phyla within soil aggregates between modern and ancient tea plantations.

Table S6 Differences of the relative abundance of the top 50 bacterial genera within soil aggregates between modern and ancient tea plantations.

Table S7 Differences of the relative abundance of the top 12 fungal phyla within soil aggregates between modern and ancient tea plantations.

Table S8 Differences of the relative abundance of the top 50 fungal genera within soil aggregates between modern and ancient tea plantations.

Table S9 Correlations of environmental factors to the tea-leaves indicators in tea plantations through the bivariate correlation analysis.

Table S10 Correlations of soil environmental factors to soil microbial community abundance and and diversity in tea plantations through the bivariate correlation analysis.

Fig. S1. The non-metric multidimensional scaling (NMDS) of soil bacterial communities in modern and ancient tea plantations of five various sampling sites, respectively.

Fig. S2. The non-metric multidimensional scaling (NMDS) of soil fungal communities in modern and ancient tea plantations of five various sampling sites, respectively.

\section{Figures}




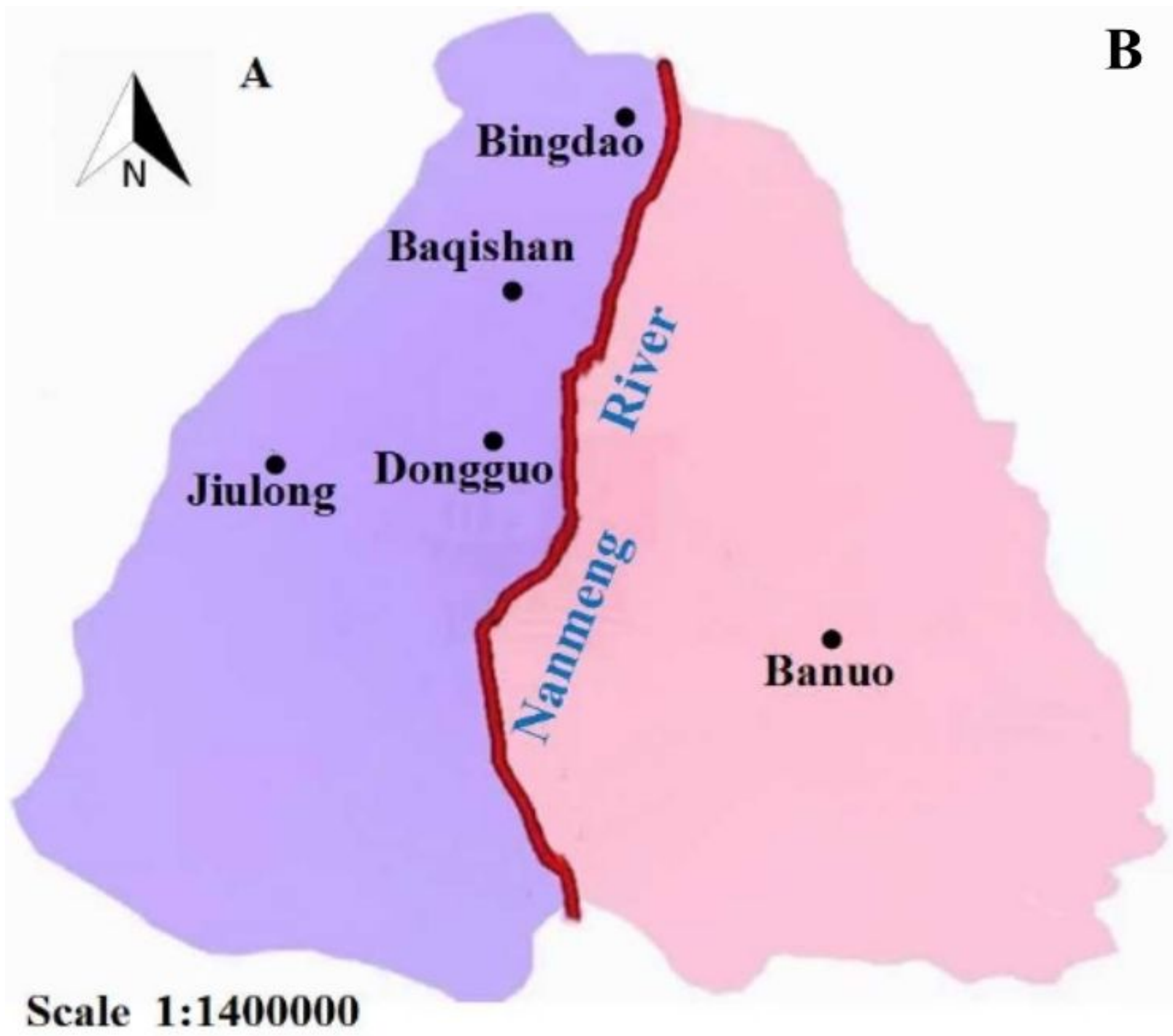

Figure 1

Geographical distribution (A) and basic situations (B) of five various sampling sites. 

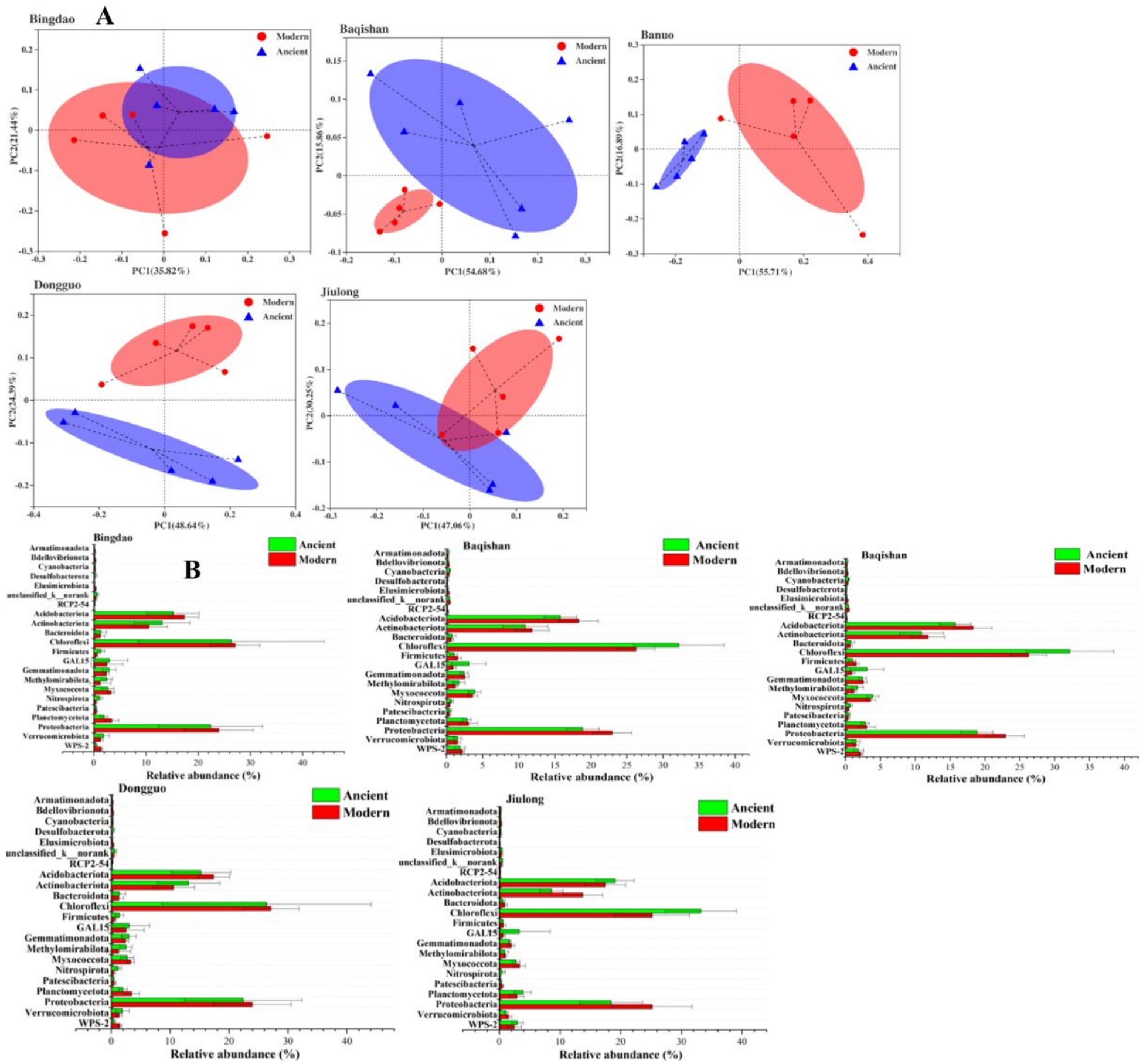

\section{Figure 2}

Principal coordinate analysis (PCoA, A) showed the remarkable difference of soil bacterial community composition between modern and ancient tea plantations in five sampling sites including Bingdao, Baqishan, Banuo, Dongguo and Jiulong, and their relative abundance differences in the top 22 phyla (B) through independent-samples T-test, respectively. 

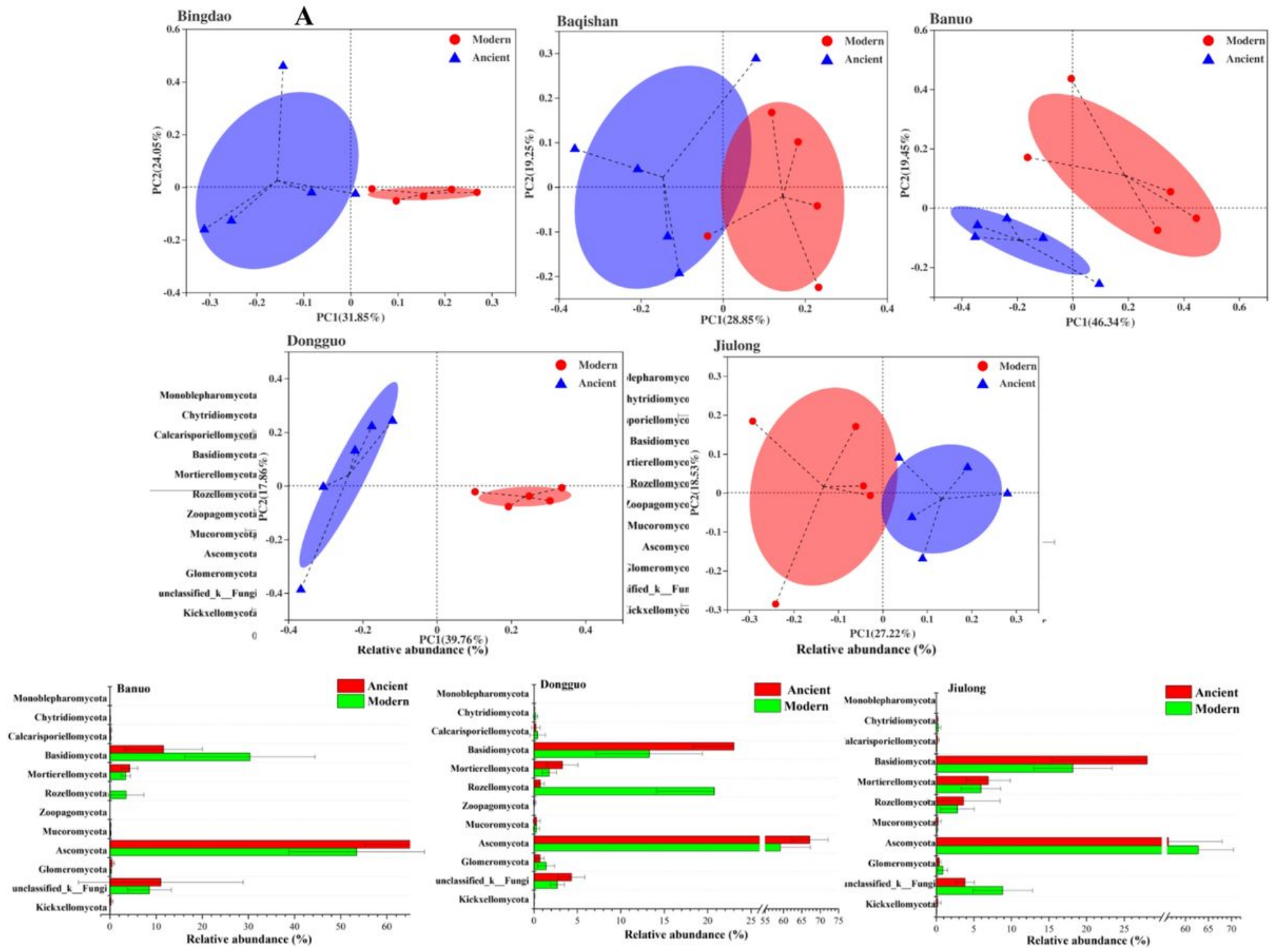

Figure 3

Principal coordinate analysis (PCoA, A) showed the remarkable difference of soil fungal community composition between modern and ancient tea plantations in five various sampling sites, and their relative abundance differences in the top 12 phyla (B) through independent-samples T-test, respectively. 

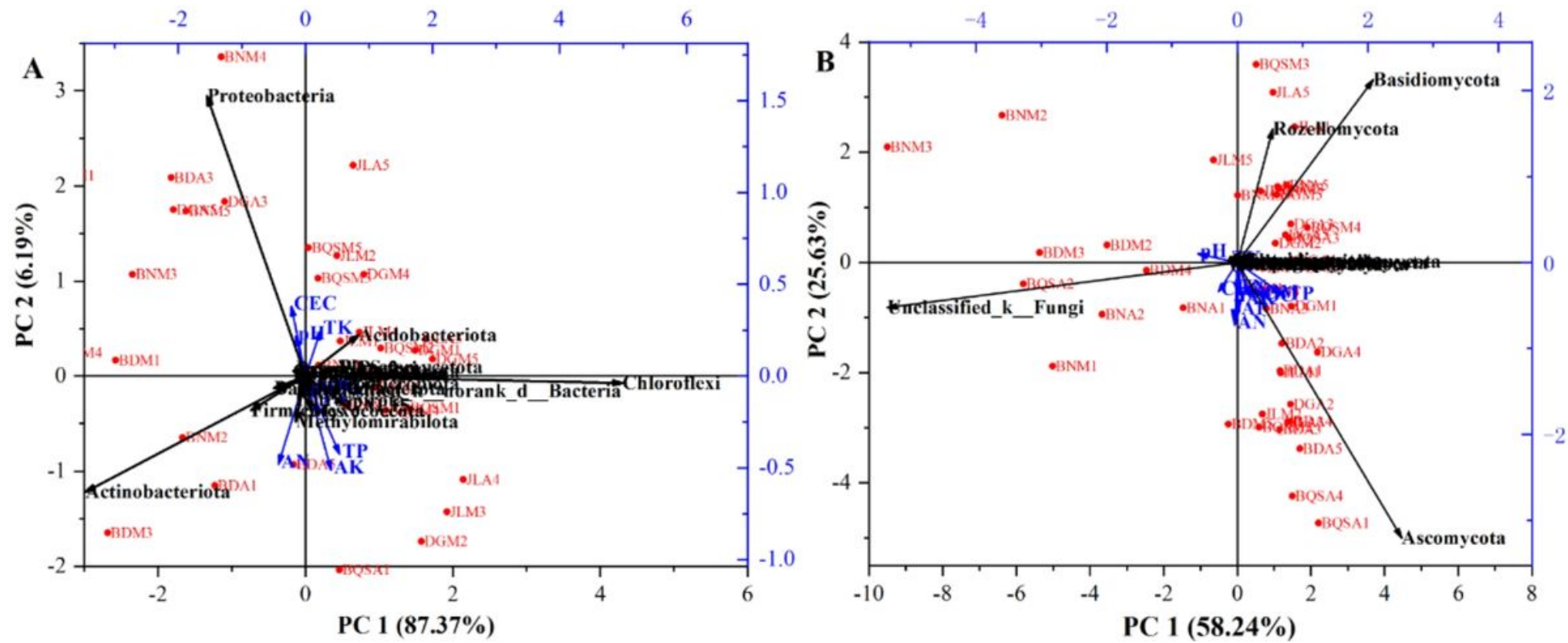

Figure 4

Internal relationships of soil environmental factors to bacterial (A) and fungal (B) community composition at the phylum level in tea plantation through redundancy analysis (RDA).

\section{Supplementary Files}

This is a list of supplementary files associated with this preprint. Click to download.

- Supportinginformation.doc 